\title{
Symptomatic Gallstones in the Young: Changing Trends of the Gallstone Disease-Related Hospitalization in the State of New York: 1996 - 2010
}

\author{
Sridhar Chilimuria, Vinaya Gaduputi ${ }^{\mathrm{a}}$, Hassan Tariq ${ }^{\mathrm{a}, \mathrm{b}}$, Suresh Nayudu ${ }^{\mathrm{a}}$, Trupti Vakde ${ }^{\mathrm{a}}$, \\ Mariela Glandt ${ }^{\mathrm{a}}$, Harish Patel ${ }^{\mathrm{a}}$
}

\begin{abstract}
Background: The aim of the study was to evaluate if the gallstonerelated hospitalizations in the young $(<20$ years of age $)$ have increased over time in both the Bronx County and New York State as a whole.
\end{abstract}

Methods: We retrospectively reviewed 15 years (1996 - 2010) of Statewide Planning and Research Cooperative System (SPARCS) data of New York State Department of Health. Patients with ICD-9 code diagnosis of 574 (cholelithiasis) among the first three discharge diagnoses were reviewed.

Results: Total number of all cause admissions to hospitals had increased from 2.44 million to 2.77 million (1996 - 2010). However, gallstone-related hospitalizations had decreased from $1.7 \%$ to $1.2 \%$. It was noted that there was a $30 \%$ increment in the proportion of those below 20 years of age with gallstone disease requiring hospitalization over the same period. This young patient population contributed only $2.04 \%$ to all gallstone-related hospitalizations in 1996, whereas it had increased to $2.96 \%$ in 2010 . This trend was more pronounced in women, Hispanics and in those who were residing in the Bronx County as compared to all other New York counties combined.

Conclusion: The gallstone-related hospitalizations in the young $(<20$ years of age) have increased over time in both the Bronx County and New York State as a whole. This could be due to increasing prevalence of risk factors such as obesity, physical inactivity, diabetes and early pregnancy.

Keywords: Gallstone in young; Gallstone and teenage pregnancy; Gallstone trend in Bronx; Teenage obesity and gallstone; Changing

Manuscript accepted for publication December 01, 2016

aDepartment of Medicine, Bronx Lebanon Hospital Center, 1650 Selwyn Ave., Suite \#10C, Bronx, NY 10457, USA

${ }^{b}$ Corresponding Author: Hassan Tariq, Department of Medicine, Bronx Lebanon Hospital Center, 1650 Selwyn Ave., Suite \#10C, Bronx, NY 10457, USA. Email: HTARIQ@bronxleb.org

doi: https://doi.org/10.14740/jocmr2847w trends of the gallstone disease

\section{Introduction}

Gallstone disease is a common problem affecting almost 15$20 \%$ of the population in the United States [1]. The disease is ranked fifth amongst all the digestive diseases listed as discharge diagnoses, though it is thought to be well underestimated [2]. Age is a major risk factor for development of gallstone disease [3]. Gallstones typically form between 20 and 40 years of age, but become symptomatic much later [4]. However, at our institution, we noticed that the proportion of teenagers undergoing endoscopic retrograde cholangiopancreatography (ERCP) has increased over time, which could be related to increased prevalence of symptomatic gallstone disease in this age group. A total of 354 patients underwent ERCP for management of symptomatic cholelithiasis from 2003 to 2010. Seventy-one percent of the patients were women. The proportion of women undergoing ERCP below 20 years of age increased from $30 \%$ in 2003 to $53 \%$ in 2010 .

It has been noted in the studies from France that the prevalence of the gallstone disease in population below 19 years of age is between $0.13 \%$ and $2 \%$ [5]. It also has been reported that the prevalence of idiopathic gallstones in the pediatric age group is more than previously thought [6]. Childhood obesity and use of oral contraceptive pills, both known to be strong independent risk factors for gallstone disease, have been steadily increasing in recent years [7]. A populationbased study from Texas found that Hispanic race along with obesity positively correlated with incidence of gallstone disease in children [8]. It also has been observed that idiopathic gallstone disease in adolescent women is more likely to cause symptoms as compared to the gallstone disease secondary to hemolytic disorders [6]. In a population study in the Bronx, teenage pregnancies were linked to the symptomatic cholelithiasis [9]. Poorest areas in New York City including South Bronx have also been cited to have highest levels of obesity [10]. The prevalence of obesity in adolescent boys and girls of Bronx was pegged at $24.9 \%$ and $20.1 \%$, respectively, by a 
cross-sectional study [11]. We studied the trends of the gallstone hospitalizations among children and adolescents in the state of New York State (NYS) and present the most recent estimates (1996 - 2010).

\section{Patients and Methods}

\section{Study population}

This is a retrospective study done with anonymous data obtained from Statewide Planning and Research Cooperative System (SPARCS) database of New York State Department of Health. Prior authorization was taken from Bureau of Biometric and health statistics for utilization of the SPARCS database. The data correspond to a period of 15 years from 1996 to 2011 .

The data were extracted from the SPARCS database using SPSS v20 for windows. The DOT file map provided by Department of Health was used to locate the parameters in the database. The discharge diagnoses in SPARCS database were identified by ICD-9 codes. The patients with ICD-9 diagnoses of 574 among any of the first three discharge diagnoses were selected. The ICD code of 574 is generic to all types of cholelithiasis and its related complications. Other demographic variables that were obtained from the database included patients' age at the time of hospitalization, gender, race and county of residence. However, the patients with co-existent diagnosis of either HIV or abortion on medical record could not be identified with their counties of residence and therefore were not included in the study. During the final analysis, the patients were attributed to their respective counties of residence without taking into account their counties of hospitalization.

\section{Study acronyms - definitions}

We used certain terms to define a particular subset of population in the study. These terms have been used in parentheses in the results section. They are as follows: 1) AD: All discharges irrespective of the age, gender and ICD codes of the discharge diagnoses. 2) U20: All discharges among patients younger than 20 years, irrespective of gender and ICD codes of the discharge diagnoses. 3) U20F: All discharges among female patients younger than 20 years, irrespective of the ICD codes of the discharge diagnoses. 4) GS: All patients with a discharge diagnosis of gallstone disease. 5) GSU20: All patients younger than 20 years and with a discharge diagnosis of gallstone disease. 6) GSU20F: Female patients younger than 20 years and with a discharge diagnosis of gallstone disease.

\section{Aims \\ The aim of the study was to trend the gallstone disease-related hospitalizations and their geographical distribution, among the young (under the age of 20 years). The study intended to calcu- late the proportion of the gallstone disease amongst the young}

(under the age of 20 years) to the total burden of the disease. These analyses were conducted separately for different geographical areas (Bronx vs. non-Bronx). We employed appropriate statistical methods to see if the trends were significantly different among individuals belonging to different age strata and counties of residence.

The study population was therefore divided into two groups: a group containing patients younger than 20 years and the other containing patients older than 20 years. The trends of the contribution of gallstone disease in the young (age $<20$ years) to the total gallstone disease burden (GSU20/GS), over the period of 15 years from 1996 to 2010, in all counties of NYS were analyzed.

\section{Statistical methods}

SPSS v. 20 and SAS were used for all analyses. All inferential analyses were set at a $95 \%$ level of significance. A random intercept linear mixed model was computed to investigate possible differences in percentages of people under 20 years of age (GSU20/GS) with gallstones according to geographic area (Bronx vs. other counties) and year (1996 - 2010). A mixed model approach allows each county to have unique intercepts and slopes to their regression lines, and accounts for correlations of measurements of outcomes within each county. The mixed model approach gives more explicit meaning to the results obtained, over a standard regression approach. One way repeated ANNOVA was used to analyze the race distribution. Duncan's multiple range test was used to analyze the difference in the proportion amongst the different race groups.

\section{Results}

The numbers reveal that there was an uptrend in the number of hospitalization $(\mathrm{AD})$ amongst patients of all age from approximately 2.47 million in 1996 to 2.77 million in 2010 (growth of $12.5 \%$ ), with a peak in 2009 with 2.82 million. These numbers however do not include people who were either pregnant or HIV positive at the time of discharge. The trend of increase in admissions remained the same even when genders were analyzed separately. There were more females admitted than men, to hospitals during every year of the study period with an average contribution of $56.81 \%$ to the total number of admissions. If all the admissions were stratified by age, it was noted that the number of under 20 years of age (U20) all cause hospitalizations decreased from 177,572 in 1996 to 148,471 in 2010 (a decrease of $16.38 \%$ ). Females on an average contributed to $52.81 \%$ of these under 20 all cause admissions.

Gallstones contributed to $1.7 \%$ of all hospitalizations (GS) irrespective of age or gender in 1996, whereas it contributed to only $1.2 \%$ in 2010 (a decrease of 30\%). A higher proportion of these gallstone hospitalizations were contributed by females $(68.1 \%$ on an average over the 15 years of study period). In gallstone-related discharges below the age of 20 year (GS20), most of the patients were in the age group of 


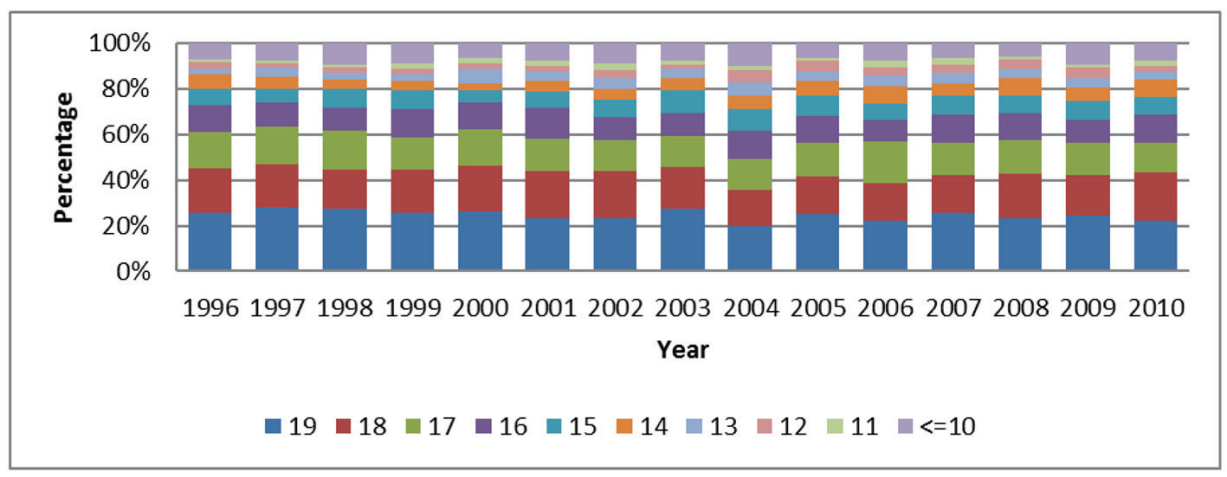

Figure 1. Age distribution in patients under the age of 20 years with gallstones (GSU20).

14 - 19 years (Fig. 1). Analysis after age-based stratification of the gallstone admissions revealed an increase in proportion of gallstone disease in patients younger than 20 years (GS20: GS), from $2.04 \%$ in 1996 to $2.96 \%$ in 2010 (Table 1). Oneway repeated measure ANOVA was performed to find out if this increase was statistically significant. The "sphericity assumed test" for the study period showed this increase to be statistically significant with $\mathrm{F}(14,140)=4.657, \mathrm{P}<0.001$. Therefore, it can be interpreted that the proportion of the below 20 gallstone admissions to total gallstone admissions (GS20/GS) was not same over the 15 years study period and each of the chronologically successive increments was statistically significant.
This trend was more pronounced in females where under 20 years (GSF20/GS) of age gallstone-related hospitalization (1.93\% of gallstone admissions and $0.04 \%$ of all female admissions) occurred at a higher percentage when compared to males $(0.4 \%$ of gallstone admissions and $0.014 \%$ of all male admissions). The relative incidence of gallstones in young females when compared to young males was 4.12:1.

There was uniform decrease in proportion of the patients admitted under 20 years of age (U20) in all counties (Fig. 2). While the proportion of the under 20 gallstone hospitalization among total gallstone admissions (i.e. GS20/GS) for NYS increased from $2.04 \%$ in 1996 to $2.96 \%$ in 2010 , in Bronx County, it increased from $3.71 \%$ in 1996 to $6.06 \%$ in 2010 (Fig.

Table 1. Age-Based Stratification of the Gallstone Admissions Showing an Increase in Proportion of Gallstone Disease in Patients Younger Than 20 Years

\begin{tabular}{llllllll}
\hline Year & $\mathbf{( A D )}^{\mathbf{i}}$ & $\mathbf{( A D - f e m a l e}^{\mathbf{i}-\mathbf{f}}$ & $\mathbf{( U 2 0}^{\mathbf{i i}}$ & $\mathbf{( G S}^{\mathbf{i i i}}$ & $\mathbf{( G S F}^{\mathbf{i i i}-\mathbf{f}}$ & (GSU20) $^{\mathbf{i v}}$ & (GSU20:GS) $^{\mathbf{v}}$ \\
\hline 1996 & $2,472,582$ & $1,420,645$ & 177,572 & 42,911 & 30,245 & 877 & $2.04 \%$ \\
1997 & $2,436,953$ & $1,398,620$ & 170,473 & 38,982 & 27,457 & 822 & $2.11 \%$ \\
1998 & $2,449,696$ & $1,407,077$ & 163,528 & 37,512 & 26,396 & 826 & $2.20 \%$ \\
1999 & $2,473,309$ & $1,417,316$ & 165,894 & 35,833 & 24,951 & 779 & $2.17 \%$ \\
2000 & $2,549,654$ & $1,460,678$ & 163,898 & 36,063 & 25,132 & 781 & $2.17 \%$ \\
2001 & $2,574,758$ & $1,470,400$ & 164,071 & 35,596 & 24,375 & 716 & $2.01 \%$ \\
2002 & $2,617,203$ & $1,493,780$ & 160,598 & 35,691 & 24,470 & 792 & $2.22 \%$ \\
2003 & $2,696,049$ & $1,536,120$ & 164,333 & 35,487 & 24,039 & 863 & $2.43 \%$ \\
2004 & $2,741,495$ & $1,556,981$ & 162,852 & 35,507 & 23,880 & 853 & $2.40 \%$ \\
2005 & $2,752,209$ & $1,554,004$ & 161,043 & 35,041 & 23,583 & 813 & $2.32 \%$ \\
2006 & $2,771,579$ & $1,563,127$ & 160,628 & 35,411 & 23,726 & 960 & $2.71 \%$ \\
2007 & $2,783,498$ & $1,571,481$ & 159,569 & 35,033 & 23,194 & 1008 & $2.88 \%$ \\
\hline 2008 & $2,809,306$ & $1,581,626$ & 154,745 & 34,100 & 22,476 & 921 & $2.70 \%$ \\
\hline
\end{tabular}

Also refer to study acronyms section. 'AD: All discharges irrespective of the age, gender and ICD codes of the discharge diag-

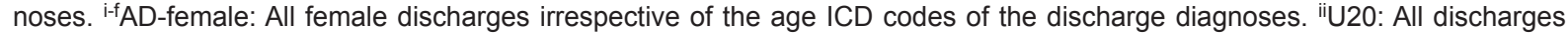
among patients younger than 20 years, irrespective of gender and ICD codes of the discharge diagnoses. iiiGS: All patients with a discharge diagnosis of gallstone disease. iii-fGSF: All female patients with a discharge diagnosis of gallstone disease. ${ }^{\text {iv } G S U 20:}$ All patients younger than 20 years and with a discharge diagnosis of gallstone disease. vPercentage of gallstone disease in the young (no. of patients aged $<20$ years with gallstone disease/total no. patients with gallstone disease). 


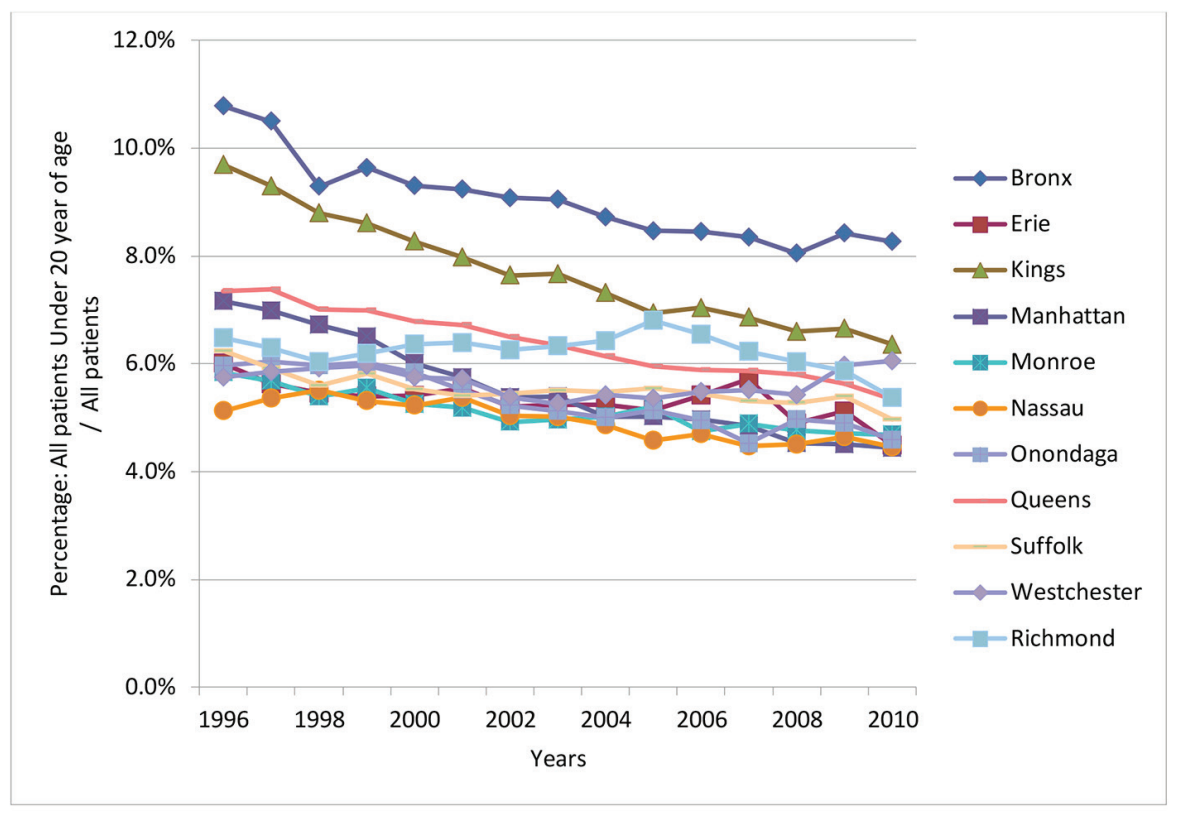

Figure 2. The decreasing proportion of the young among the all-cause hospitalizations in most of NY counties.

3). Graphic presentation of 11 counties was done because it represented $75 \%$ of the New York Population. These numbers were $1.93 \%$ in 1996 to $2.7 \%$ in 2010 , respectively, for those residing New York counties barring the Bronx. The estimated marginal means of percentages of people under 20 years of age with gallstones (GS20) for Bronx County vs. all other counties included in the study, according to year, are presented in Figure 4. It can be seen that Bronx County yearly proportion of people under 20 years of age increase (GS20/GS) over time, while the percentages of all other counties combined remain more uniform across time. The scatter plot of all data points representing percentage values for Bronx County vs. other counties according to year of study is presented in Figure 5. A linear line was fit to each group. It can be seen from the fit lines that Bronx County has a higher intercept with a slope increasing over time, while the intercept is lower for other counties and the slope is near zero.

The study population was divided into Hispanics, Blacks, Whites and "others" based on the racial distribution. There was no significant difference in the rates of hospitalization in NYS (AD) amongst any of these racial groups over the study period of 15 years. The contribution by Hispanics in Bronx towards gallstone (GS-Bronx)-related hospitalizations increased from $33 \%$ to $49 \%$, whereas the contribution from those under 20

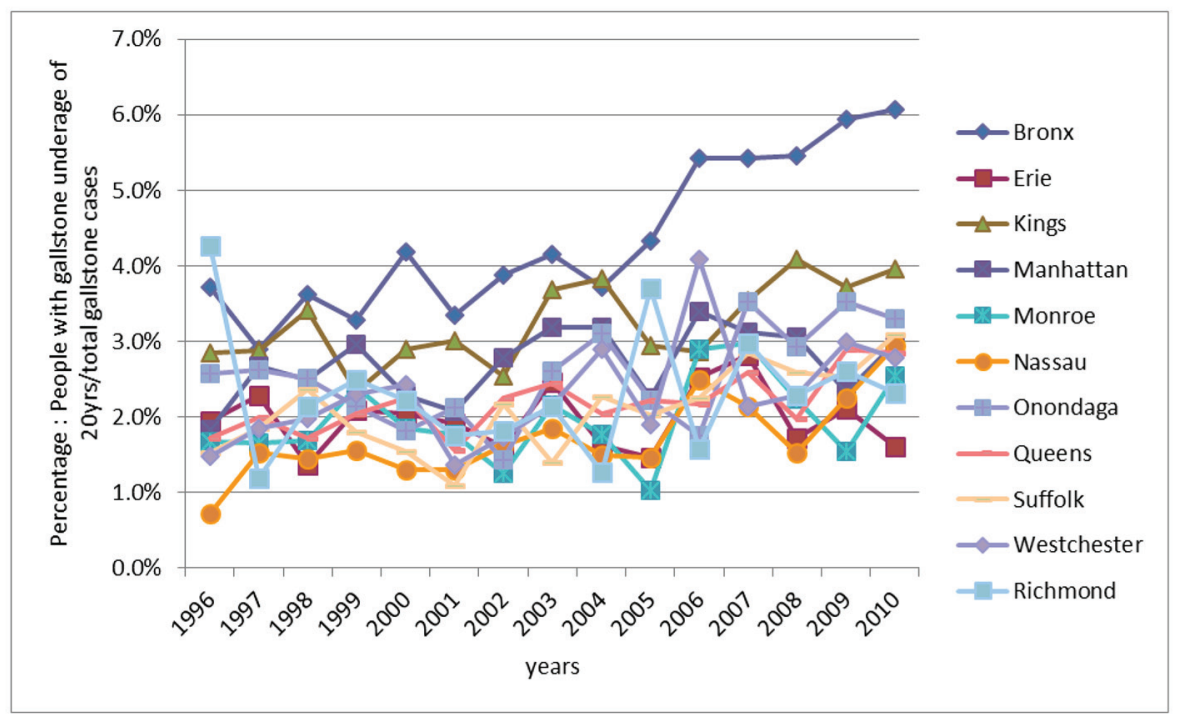

Figure 3. The increasing incidence of gallstone disease in the young $(<20$ years), with consistently highest incidence in Bronx County. 


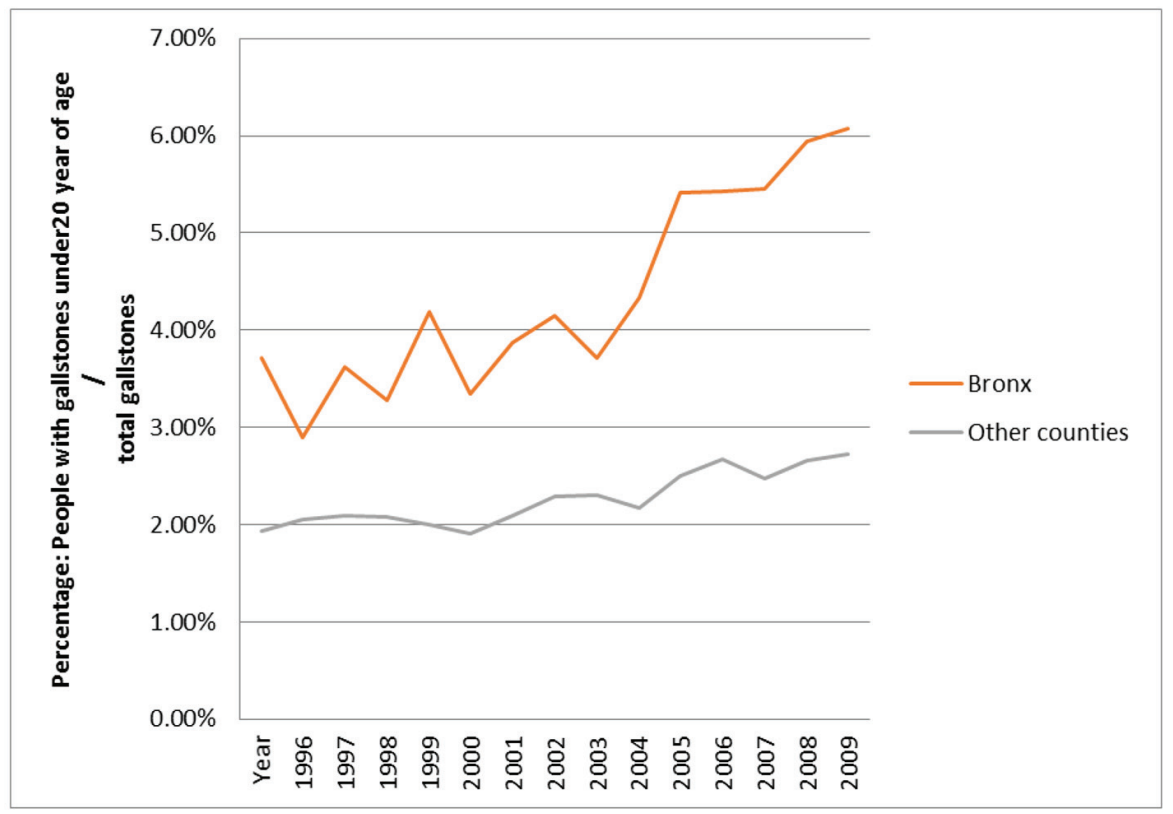

Figure 4. Estimated marginal means of percentage of patients with under the age of 20 years with gallstones for Bronx County versus other counties according to year graph.

years (GU20-Bronx) increased from 34\% to $63 \%$ (Table 2).

\section{Discussion}

Our study confirmed our initial observation that there is a marked increase in the number of patients below 20 years of age hospitalized for symptomatic gallbladder disease in the Bronx. While the total number of hospitalization for teenagers in the Bronx has decreased in the last 15 years, the percentage of patients presenting with gallstone disease has been dramatically increasing. The increase in gallstones in the younger

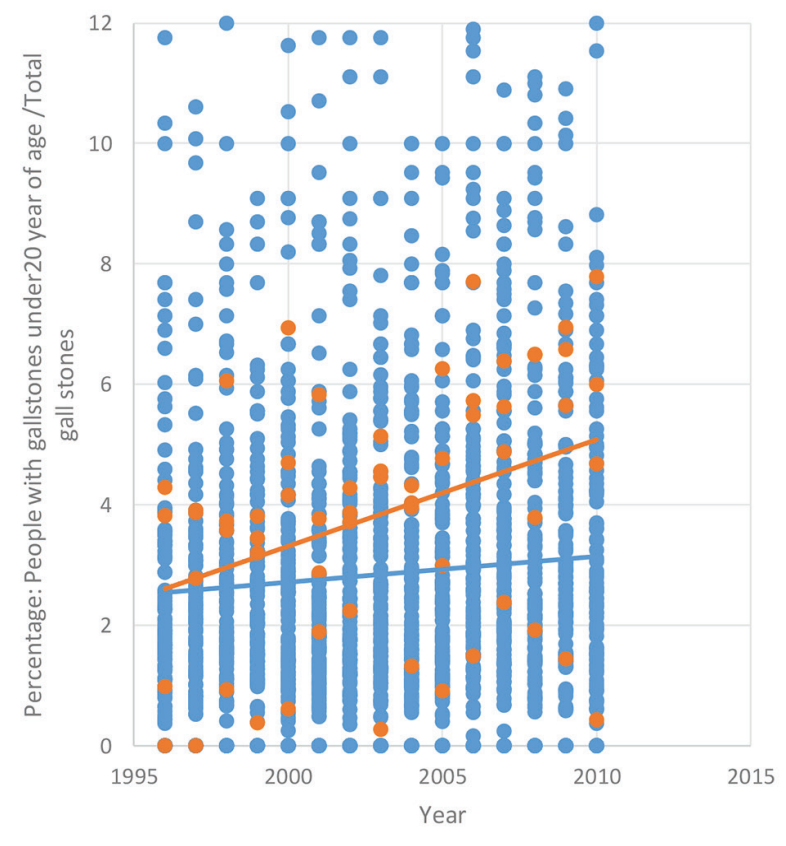

- Other Counties

- Bronx Counties

Figure 5. Scatter plot of percentages of people under 20 years of age according to county group of Bronx County vs. other counties according to year of study. 
Table 2. Contribution of Various Ethnic Groups to All Hospitalizations, All Gallstone Related Admissions and Under the Age 20 Gall Stone Related Admissions

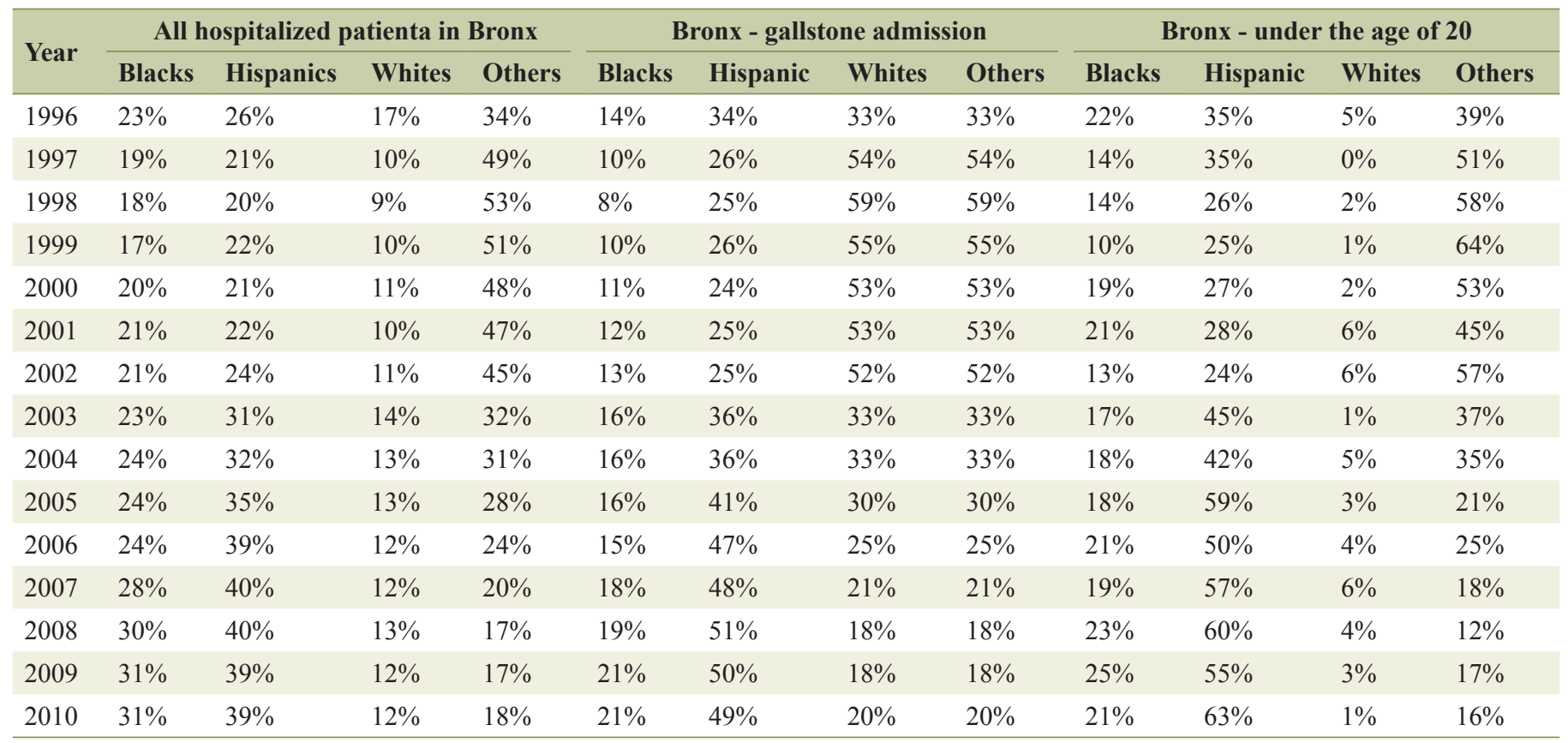

population has been observed throughout the state, but to a much lesser extent than in the Bronx.

Pregnancy is a well-known risk factor for gallstone disease. The risk is related to both the frequency and number of pregnancies, as shown in a previous study that showed that the prevalence of gallstones increased from $1.3 \%$ in nulliparous females to $12.2 \%$ in multiparous females [12]. While our data did not include what percentage of the teenagers with gallstones had a history of pregnancy, it could be postulated that it is a significant contributor to the rise in gallstones and could help explain the difference between the Bronx and other counties, given that the teen pregnancy rate in the Bronx is the highest in the state and twice the average of the 10 other counties we examined $[13,14]$. Figure 1 shows that the percentage of gallstone patients at the age of 17 - 19 years accounts for about $50-60 \%$ of all under the age of 20 patients, which can be attributed to teenage pregnancy, leading to this disease.

Teenage obesity is another important factor that possibly contributes to increase in gallstone disease. Ethnic minorities and low socioeconomic status (SES) groups, which comprise a substantial proportion of Bronx population, are traditionally at risk for increased obesity $[15,16]$. The risk of developing severe obesity (BMI $>$ 99th percentile for age and sex) is as much as 1.4-fold higher in ethnic minority adolescents [16]. It has been noted in population surveys that one in every four children in New York City public elementary schools is obese [17]. These trends seem to continue well into teenage as studies done in adolescent public school-going population in Bronx showed that prevalence of obesity was as high as $22.5 \%$ [11].

Race seems to be an independent risk factor for gallstones, with Hispanics being at a higher risk for gallstones than the rest of the population [18]. Hispanics account for 54\% of the population in the Bronx [19], which is significantly higher than the rest of the state. Of the other counties we analyzed, the second highest was Queens, with a Hispanic population of $27 \%$.

The increase in symptomatic gallstones that is being observed has several clinical implications. Firstly, it warrants procedures, such as endoscopic sphincterotomy and laparoscopic cholecystectomy, earlier in life. A Japanese study which followed 382 patients after sphincterotomy for 10 years showed that $10 \%$ of patients will develop complications in the form of stone recurrence or acute cholangitis [20]. Furthermore, this earlier presentation is concerning in light of the recent findings that in the US population, persons with gallstone disease have increased mortality overall and increased mortality from cardiovascular disease and cancer [21].

Our study is limited by the fact that it is an observational study. A large, prospective study is needed in order to further understand the changing epidemiology of symptomatic gallstone disease.

\section{Disclosures}

None.

\section{Author Contributions}

Sridhar Chilimuri conceptualized the study protocol and methodology, and performed critical revision of the final manuscript. Vinaya Gaduputi, Hassan Tariq, Suresh Nayudu, Trupti Vakde, and Mariela Glandt contributed towards writing the final manuscript. Harish Patel conceptualized the study protocol 
and methodology, worked on gathering and interpretation of data, prepared the framework for final manuscript, and modified the finalized manuscript as per the Red Journal requirements. All authors have made contributions to the article and have reviewed it before submission.

\section{Informed Consent}

Patients were not required to give informed consent for the study because the analysis used anonymous clinical data of SPARCS (Statewide Planning and Research Cooperative System) data of New York State Department of Health.

\section{Conflicts of Interest}

None of the authors have any financial conflicts of interest.

\section{References}

1. Shaffer EA. Gallstone disease: Epidemiology of gallbladder stone disease. Best Pract Res Clin Gastroenterol. 2006;20(6):981-996.

2. Everhart JE, Ruhl CE. Burden of digestive diseases in the United States part I: overall and upper gastrointestinal diseases. Gastroenterology. 2009;136(2):376-386.

3. Barbara L, Sama C, Morselli Labate AM, Taroni F, Rusticali AG, Festi D, Sapio C, et al. A population study on the prevalence of gallstone disease: the Sirmione Study. Hepatology. 1987;7(5):913-917.

4. Sparkman RS. Gallstones in young women. Ann Surg. 1957;145(6):813-824.

5. Debray D, Franchi-Abella S, Irtan S, Girard M. [Cholelithiasis in infants, children and adolescents]. Presse Med. 2012;41(5):466-473.

6. Herzog D, Bouchard G. High rate of complicated idiopathic gallstone disease in pediatric patients of a North American tertiary care center. World J Gastroenterol. 2008;14(10):1544-1548.

7. Koebnick C, Smith N, Black MH, Porter AH, Richie BA, Hudson S, Gililland D, et al. Pediatric obesity and gallstone disease. J Pediatr Gastroenterol Nutr. 2012;55(3):328-333.

8. Mehta S, Lopez ME, Chumpitazi BP, Mazziotti MV,
Brandt ML, Fishman DS. Clinical characteristics and risk factors for symptomatic pediatric gallbladder disease. Pediatrics. 2012;129(1):e82-88.

9. Shafer AD, Ashley JV, Goodwin CD, Nanagas VN, Jr., Elliott D. A new look at the multifactoral etiology of gallbladder disease in children. Am Surg. 1983;49(6):314319.

10. Wallach JB, Rey MJ. A socioeconomic analysis of obesity and diabetes in New York City. Prev Chronic Dis. 2009;6(3):A108.

11. Isasi CR, Whiffen A, Campbell E, Florez Y, Freeman K, Wylie-Rosett J. High prevalence of obesity among innercity adolescent boys in the Bronx, New York: forgetting our boys. Prev Chronic Dis. 2011;8(1):A23.

12. Valdivieso V, Covarrubias C, Siegel F, Cruz F. Pregnancy and cholelithiasis: pathogenesis and natural course of gallstones diagnosed in early puerperium. Hepatology. 1993;17(1):1-4.

13. Bronx County Teen pregnancy rate per 1,000 females aged 15-19 years. 2013 [cited; Available from: https:// www.health.ny.gov/statistics/chac/birth/b13_58.htm.

14. Teen Births. 2012 [cited; Available from: http://www.cdc. gov/nchs/fastats/teen-births.htm.

15. Wang Y, Zhang Q. Are American children and adolescents of low socioeconomic status at increased risk of obesity? Changes in the association between overweight and family income between 1971 and 2002. Am J Clin Nutr. 2006;84(4):707-716.

16. Ogden CL, Carroll MD, Flegal KM. High body mass index for age among US children and adolescents, 20032006. JAMA. 2008;299(20):2401-2405.

17. Thorpe LE, List DG, Marx T, May L, Helgerson SD, Frieden TR. Childhood obesity in New York City elementary school students. Am J Public Health. 2004;94(9):1496-1500.

18. Everhart JE. Gallstones and ethnicity in the Americas. J Assoc Acad Minor Phys. 2001;12(3):137-143.

19. Bureau USC. State and County Quickfacts 2014 [cited 2014 July 2014]; Available from: http://quickfacts.census.gov/qfd/states/36/36005.html.

20. Sugiyama M, Atomi Y. Follow-up of more than 10 years after endoscopic sphincterotomy for choledocholithiasis in young patients. Br J Surg. 1998;85(7):917-921.

21. Ruhl CE, Everhart JE. Gallstone disease is associated with increased mortality in the United States. Gastroenterology. 2011;140(2):508-516. 